\title{
Neurological and neuropsychological functions in adults with a history of developmental arsenic poisoning from contaminated milk powder
}

\section{Citation}

Yorifuji, Takashi, Tsuguhiko Kato, Hitoshi Ohta, David C. Bellinger, Kenichi Matsuoka, and Philippe Grandjean. 2016. "Neurological and Neuropsychological Functions in Adults with a History of Developmental Arsenic Poisoning from Contaminated Milk Powder." Neurotoxicology and Teratology 53 (January): 75-80. doi:10.1016/j.ntt.2015.12.001.

\section{Published Version}

10.1016/j.ntt.2015.12.001

\section{Permanent link}

http://nrs.harvard.edu/urn-3:HUL.InstRepos:37221723

\section{Terms of Use}

This article was downloaded from Harvard University's DASH repository, and is made available under the terms and conditions applicable to Open Access Policy Articles, as set forth at http:// nrs.harvard.edu/urn-3:HUL.InstRepos:dash.current.terms-of-use\#OAP

\section{Share Your Story}

The Harvard community has made this article openly available.

Please share how this access benefits you. Submit a story.

Accessibility 
Neurological and neuropsychological functions in adults with a history of developmental arsenic poisoning from contaminated milk powder

Takashi Yorifuji MD $\mathrm{MD}^{\mathrm{a}}$, Tsuguhiko Kato $\mathrm{PhD}^{\mathrm{b}}$, Hitoshi Ohta $\mathrm{MD}^{\mathrm{c}}$, David C. Bellinger $5 \quad \mathrm{PhD}, \mathrm{MSc}^{\mathrm{d}, \mathrm{e}}$, Kenichi Matsuoka $\mathrm{MD}^{\mathrm{c}}$, Philippe Grandjean $\mathrm{MD}^{\mathrm{e}, \mathrm{f}}$

${ }^{a}$ Department of Human Ecology, Graduate School of Environmental and Life Science, Okayama University, Okayama, 700-8530, Japan

${ }^{\mathrm{b}}$ Department of Social Science, National Center for Child Health and Development,

10 Tokyo, 157-8535, Japan

${ }^{c}$ Mizushima Kyodo Hospital, Kurashiki, Okayama, 712-8567, Japan

${ }^{\mathrm{d}}$ Department of Neurology, Children's Hospital, Harvard Medical School, Boston, MA 02115, USA.

${ }^{\mathrm{e}}$ Department of Environmental Health, Harvard T.H.Chan School of Public Health,

15 Boston, MA 02215, USA

${ }^{\mathrm{f}}$ Department of Environmental Medicine, University of Southern Denmark, DK-5000, Odense, Denmark

\section{Corresponding author:}

20 Takashi Yorifuji

Department of Human Ecology, Graduate School of Environmental and Life Science, Okayama University, 3-1-1 Tsushima-naka, Kita-ku, Okayama 700-8530, Japan Tel: +81-86-251-8925; E-mail: yorichan@md.okayama-u.ac.jp 
Running title: Neurological and neuropsychological consequences of arsenic

Contents: 291 words for abstract, 3,052 words for main text, 34 references, and 8 tables 


\begin{abstract}
During the summer of 1955 , mass arsenic poisoning of bottle-fed infants
\end{abstract} occurred in the western part of Japan due to contaminated milk powder, and more than 100 died; some childhood victims were later found to suffer from neurological sequelae

5 in adolescence. This unique incident enabled us to explore infancy as a critical period of arsenic exposure in regard to developmental neurotoxicity and its possible persistence through adulthood. The purpose of this work is to evaluate the association between developmental arsenic exposure and the neurological outcomes more than 50 years later. We conducted a retrospective cohort study during the period from April 2012 to 10 February 2013 in two hospitals in Okayama Prefecture, Japan. The study sample consisted of 50 individuals: 27 known poisoning victims from Okayama Prefecture, and 23 non-exposed local controls of similar age. In addition to neurological examination, we adapted a battery of neurophysiological and neuropsychological tests to identify the types of brain functions affected by early-life arsenic exposure. While limited

15 abnormalities were found in the neurophysiological tests, neuropsychological deficits were observed. Except for Finger tapping, all test scores in the exposed group Vocabulary and Block Design from Wechsler Adults Intelligent Scale III, Design memory subtest from Wide Range Assessment of Memory and Learning 2, and Grooved pegboard test - were substantially below those obtained by the unexposed. The exposed group showed average performance at least 1.2 standard deviations below the average for the controls. Exposed participants performed less well than controls, even after exclusion of subjects with recognized disabilities or those with a high level of education. Adults who had suffered arsenic poisoning during infancy revealed neuropsychological dysfunctions, even among those subjects not recognized as having 
disabilities. Developmental neurotoxicity due to arsenic likely results in permanent changes in brain functions.

\section{Keywords:}

5 Arsenic; Food contamination; Milk substitute; Neurological examinations;

Neurophysiological Monitoring; Neuropsychological Tests

\section{Abbreviations:}

BAEP: Brainstem auditory evoked potential

10 NCV: Nerve conduction velocity

VEP: Visual evoked potential

WRAML2: Wide Range Assessment of Memory and Learning 2

WAIS-III: Wechsler Adults Intelligence Scale III 


\section{Introduction}

Chronic exposure to inorganic arsenic via inhalation or drinking-water ingestion has been convincingly linked to lung, skin, and urinary bladder cancer [18]. In addition, arsenic is known to cause neurotoxicity [10], and recent studies suggest that

5 early-life exposure to arsenic can cause developmental neurotoxicity $[17,21,23]$. However, most previous studies were cross-sectional [14,22,24-29,32] and only a few studies were longitudinal $[3,4,20]$, thus leaving the relation of adverse effects to the timing of exposure uncertain. Therefore, little is known about the possible variation in susceptibility depending on age, i.e., the critical period of exposure.

During the summer of 1955 , mass arsenic poisoning of bottle-fed infants occurred in the western part of Japan due to contaminated milk powder that resulted in an estimated arsenic concentration in the reconstituted milk of about 4-7 mg/L [1,5]. The contaminated milk was announced as the cause of the poisoning cases and was recalled on August $24^{\text {th }} 1955$. At that point, exposures had lasted for at least four

15 months since the change in formulation of the milk powder in April 1955 [5]. Approximately 13,000 Japanese children were affected, and most cases occurred in the western part of the country where the factory was located [7]. Affected infants manifested skin pigmentation, diarrhea, and fever, and more than 100 died from acute poisoning [5]. Subsequent studies showed that adolescents exposed as infants to the 20 contaminated milk powder had a lower IQ than the general population $[12,33]$.

This unique incident of arsenic exposure enables us to explore infancy as a critical period of arsenic exposure in regard to developmental neurotoxicity persisting through adulthood. Therefore, we aimed to evaluate the association between developmental arsenic exposure from contaminated milk powder and the neurological, 
neurophysiological, and neuropsychological outcomes more than 50 years later. The overall purpose was to identify the types of brain functions primarily affected by early-life arsenic exposure.

\section{2. Methods}

\subsection{Study setting}

We conducted a retrospective cohort study during the period from April 2012 to February 2013 at two hospitals (Okayama Kyoritsu Hospital and Mizushima Kyodo Hospital) in Okayama Prefecture, Japan. We targeted Okayama Prefecture because this

10 Prefecture was the most severely affected area in the country [5]. We determined neurological, neurophysiological, and neuropsychological outcomes as well as demographic characteristics using questionnaires in connection with the participants' regular health examinations. All examiners were blinded as to the participants' exposure status.

15

\subsection{Study participants}

The study sample consisted of 50 individuals who provided written, informed consent for participation in the study: 27 among known poisoned victims in Okayama Prefecture and 23 local, non-exposed controls. Among those invited, three exposed and

20 three non-exposed individuals refused to participate in the study. The exposed participants were identified from a list of victims known to the Hikari Association to receive free health care at the two hospitals $(n=15)$. The Hikari Association is a public-interest foundation dedicated to provide permanent relief for the victims of the contaminated milk powder. It supports victims through various efforts, such as benefits, 
including living allowance, and regular health examinations for those who have disabilities or a relevant medical diagnosis. According to the Hikari Association, it supports regular health examinations for victims with disorders specific to arsenic poisoning, such as intellectual disability, mental disorder, or skin lesions.. Among the

515 participants identified through the Association, 12 received benefits for their disorders, while three participants did not receive any, because they did not suffer from qualifying disorders. The list of exposed participants was supplemented by a list of victims who did not receive regular health examinations offered by the Hikari Association but who could be reached through the two local hospitals $(n=12)$; the

10 hospitals offered health care to these victims regardless of their current health status. Thirteen exposed participants were born in 1954 and were at least five months old when the contamination started, while 14 were born in 1955 and would therefore have been exposed at a younger age before the official warning against the milk powder was issued in August of 1955. No information could be retrieved about the duration of

15 breast-feeding. We recruited 23 non-exposed controls from the medical staff, including clerks, nurses, laboratory technicians, and one physician at the two hospitals, at ages similar to the exposed participants. None of them had been exposed to the contaminated milk powder during infancy.

\subsection{Neurological tests}

One neurologist employed at each of the two hospitals conducted all clinical neurological examinations. They performed a screening-type neurological examination including mental status (e.g., level of consciousness, speech and language, memory, agnosia, and apraxia), cranial nerves, motor system (e.g., visual inspection, tone, and 
strength), sensory system (e.g., touch, pain, vibration, and joint position sense), reflexes, coordination (e.g., finger-nose-finger), and gait. They also asked the participants about symptoms related to autonomic function (e.g., genitourinary retention, incontinence, constipation, and diarrhea) and checked for orthostatic hypotension. Based on these

5 results, they provided summary scores with regard to mental, motor, sensory, and autonomic functions being normal or abnormal.

\subsection{Neurophysiological tests}

All of the neurophysiological tests [Nerve conduction velocity (NCV),

10 brainstem auditory evoked potentials (BAEPs), and visual evoked potentials (VEPs)] were conducted in the hospitals using standardized methods and the same instrumentation (Neuropack S1, Nihon Kohden). We calculated motor NCV of the median nerve in both hands. We performed BAEPs and VEPs on both sides and used the averages for statistical analyses. Because VEP assessment was not possible in one

15 hospital (Okayama Kyoritsu Hospital), only the 23 participants at the other hospital were tested. Although pattern-reversal VEPs may be preferable because of their better specificity, we used flash VEPs due to its availability as routine procedure.

\subsection{Neuropsychological tests}

One psychologist (TK) administered all of the neuropsychological tests to all participants. The tests took about half an hour on average, although exposed subjects generally took longer than the controls. 
2.5.1. Two subtests (Vocabulary and Block Design) from Wechsler Adults Intelligent Scale III (WAIS-III)

WAIS-III is a test for measuring intelligence in adults and contains 14 subtests. Out of those, we selected two (Vocabulary and Block Design). With regard to

5 Vocabulary, words of increasing difficulty were presented to the participants both orally and visually, and they were asked to define the words. This component measures verbal knowledge and concept formation. With regard to Block Design, the participants were asked to arrange the blocks to match the design formed by the examiner or shown on cards. This component measures spatial problem-solving and manipulative abilities. We

10 then calculated standardized scores of both components.

2.5.2. Design memory subtest from Wide Range Assessment of Memory and Learning 2 (WRAML2)

WRAML2 measures learning and memory functions and is composed of six

15 core subtests. We selected design memory subtest among them. The participants viewed each card for five seconds, and then after a ten-second delay the participants were asked to draw what they remembered. The participants repeated the task five times for five stimulus cards. The subtest measures reproduction from visual memory.

2.5.3. Grooved pegboard test

Grooved pegboard is a dexterity test to measure complex visual-motor coordination. We used the Grooved Pegboard (Model 32025, Lafayette Instrument Company, USA), which consists of 25 holes with randomly positioned slots. The participants were asked to rotate pegs, which have a key along one side, to match the 
hole. The participants performed the test with both dominant and non-dominant hands. We recorded the duration (in seconds) required to perform each trial, beginning when the participants started the task and ending when the last peg was correctly placed.

\section{$5 \quad$ 2.5.4. Finger tapping test}

Finger tapping measures maximum oscillation rate of the index finger, which can act as an indicator of psychomotor disruption. The participants were asked to tap the circular paddle attached to a counter as rapidly as possible in a ten-second trial period and the number of times the participants tapped was recorded. If five consecutive scores

10 within a range of five points were obtained, we calculated the mean of those five scores for the final score. But, if not, we administered ten trials and calculated the mean of the five highest scores for the final score. We used Finger Tapper (Model 32726, Lafayette Instrument Company, USA).

15 2.6. Statistical analyses

We compared descriptive characteristics between exposed and unexposed participants. We also compared neurological, neurophysiological, and neuropsychological outcomes between them. We used chi-square tests for binary outcomes and t-tests for continuous outcomes. To the extent that demographic 20 characteristics significantly differed between exposed and unexposed participants, we performed multivariate analysis adjusting for the relevant parameters. However, we did not adjust for parameters considered to be intermediate variables between arsenic exposure and outcomes or possible outcomes of the exposure (e.g., education or 
employment status). With regard to the Grooved pegboard and Finger tapping tests, we compared the outcomes for dominant and non-dominant hands.

As a sensitivity analysis, we repeated the analyses after exclusion of 12 exposed participants who received regular health examinations offered by the Hikari

5 Association (due to the severity of their disorders). We also repeated the analyses after excluding the participants who had been identified by the neurologists as having abnormal mental functions. In a sensitivity analysis, we also excluded participants with higher education, mainly from the control group, and repeated the analysis. We further compared outcomes between the exposed participants born in 1954 and those born in 101955.

PASW Statistics software (SPSS Japan Inc., version 18.0J) was used for the analyses. We report two-sided p-values, and those less than 0.05 were considered significant.

15 2.7. Ethical issues

This study was approved by the Institutional Review Boards of Okayama University (No. 487) and at each hospital. All of the participants provided written informed consent.

\section{3. Results}

Demographic characteristics by exposure status are shown in Table 1. While age or sex were not significantly different, the unexposed participants were more likely to have higher education or to be employed. 
Table 2 shows the summarized neurological findings. The exposed participants were more likely to have problems related with mental function, motor function, and autonomic function than the unexposed participants, but the difference was statistically significant only for mental function. The mental problems identified by the neurologists

5 included schizophrenia $(n=3)$, depression $(n=1)$, and intellectual disability $(n=2)$.

With regard to neurophysiological findings (NCVs, BAEPs, and VEPs), we did not observe any clear difference between the groups, although some latencies, especially in VEP, tended to be longer in the exposed group (Table 3). However, the exposed participants had more unfavorable neuropsychological outcomes than the 10 unexposed participants (Table 4). All of the scores except for Finger Tapping were significantly lower or longer among the exposed than the unexposed. Most of the means and standard deviations (SDs) of the controls were close to expectation. Thus, scaled scores are based on a mean of 10 with a SD of 3 for the WAIS-III subtests [30] and Design memory [16], while expected values were 68.10 (9.42) for the dominant hand 15 and $74.70(10.51)$ for the non-dominant hand in the Grooved pegboard tests [9]. However, the exposed group showed averages that were at least 1.2 SDs below the average for the controls. Likewise, the SDs of the exposed group were much greater for all tests, except for Finger Tapping.

After excluding 12 exposed participants who received regular health 20 examinations offered by the Hikari Association, no significant difference in mental status was observed and the neurophysiological findings were consistent with the original analysis (data not shown). However, the exposed participants still had significantly unfavorable outcomes on the two WAIS-III subtests and on Grooved 
pegboard (Table 5). The means differed by at least $0.7 \mathrm{SD}$, and the variability within the exposed group remained high.

In addition, even after excluding six participants identified by the neurologists as having abnormal mental functions, the exposed participants still had unfavorable

5 outcomes on the neuropsychological tests (Table 6). Again, the means of the two groups differed by at least $0.7 \mathrm{SD}$.

When we excluded participants with high educational attainment (i.e., university or higher) ( $\mathrm{n}=3$ for the exposed and $\mathrm{n}=9$ for the unexposed), the exposed participants still had unfavorable outcomes on neuropsychological tests (Table 7) and

10 the means of the two groups differed by at least $1.2 \mathrm{SD}$.

Finally, the exposed participants born in 1955 and 1954 did not differ in regard to outcomes including neuropsychological performance (data not shown). However, after excluding six participants identified by the Hikari Association to have abnormal mental functions, the exposed participants born in 1954 tended to have more unfavorable neuropsychological outcomes than those born in 1955, and the variability within the former group remained high (Table 8).

\section{Discussion}

The Morinaga milk poisoning episode affected thousands of victims, most of

20 whom were infants who received the arsenic contamination from bottle feeding. More than 100 died from acute poisoning, and neurological sequelae were observed in severe cases at adolescence. Very limited follow-up has been accomplished, although elevated mortality from nervous system disease, respiratory disease, circulatory disease, external causes, and cancer were reported among the victims $[19,34]$. The present study reports 
for the first time the neuropsychological functioning of victims at adult age. While limited abnormalities were found on the neurophysiological tests, neuropsychological deficits were apparent and remained significant after exclusion of participants with known disabilities or abnormal mental function.

Although inorganic arsenic poisoning is known to cause peripheral neuropathy $[10,31]$, we observed no abnormalities in nerve conduction velocity (NCV). This could be because we only assessed motor and not sensory NCV. However, a likely explanation is that arsenic exposure during early infancy may particularly target central nervous functions rather than peripheral nerves that are known to be particularly

10 vulnerable in adults [31]. Indeed, increased tendon reflexes rather than peripheral neuropathy, and absence of abnormalities in electromyogram were reported among the infant victims in the early phase of the milk poisoning [5].

The types of neuropsychological deficits observed are consistent with previous studies of young subjects $[12,21,33]$. The present study suggests that multiple brain

15 functions are negatively affected by the exposure, and it therefore seems plausible that arsenic exposure during early infancy may cause multi-domain or perhaps diffuse dysfunctions, and that the deficits are permanent, as one might anticipate in regard to developmental neurotoxicity [2]. Thus, the brain's sensitivity to arsenic may change during infancy and childhood, and exposures at later ages may target more specific 20 functions, with exposures at adult age primarily affecting the peripheral nervous system. When the poisonings happened, the professional opinion at the time suggested that the victims would completely recover once the clinical symptoms faded. The present study documents that deficits persist at advanced age; at least through the late fifties. This finding is in accordance with the current notion that developmental 
neurotoxicity is likely to be permanent, as has been shown in regard to arsenic $[17,21,23]$ as well as other neurotoxicants, such as alcohol, lead, and methylmercury $[2,6,11,15]$.

The number of subjects examined is small because we only recruited victims

5 living in Okayama Prefecture, but statistical significance was achieved in regard to several outcomes. Despite the small number of subjects, the study sample likely represents the patient population that could be reasonably identified in the local community (e.g., Okayama Prefecture). Apart from the Hikari Association's register of victims in need of support, no general listing is available to identify victims today. Our

10 approach to inviting Morinaga milk poisoning victims therefore represents a feasible and reproducible means of selecting subjects with severe arsenic exposure during infancy. Still, many victims were probably not known to the hospitals, especially milder cases. However, we nonetheless observed unfavorable neuropsychological outcomes among the exposed participants even after excluding the 12 subjects with severe

15 disabilities. Further, the healthy control group was not ideal, as hospital staff may not represent the general background population, as reflected by the average WAIS-III scores. However, other outcome parameters (i.e., Design memory and Grooved pegboard tests) were close to expectation for healthy subjects, and the differences in neuropsychological performance would be difficult to ascribe only to higher educational

20 achievement of the controls as shown in the sensitivity analysis excluding those with the highest level of education (Table 7). Due to the early-life exposure of the poisoning victims and its cognitive consequences, further adjustment for education would be inappropriate. 
We do not have information about the duration of breast-feeding of the participants. As bottle-feeding was heavily promoted at the time, some infants probably received bottled milk from early age, perhaps along with breast milk. It is therefore not possible to distinguish any protective effects of breast-feeding on neurocognitive

5 functions [8] in regard to the adverse effects of the contaminated milk powder, although confounding from breast-feeding would clearly be insufficient to explain the deficits observed in the exposed subjects.

Other limitations include the lack of information on the total dose of arsenic among the exposed participants. Any dose calculation is virtually impossible, because

10 the arsenic concentration differed by lot number [1]. With an estimated arsenic concentration in milk made from the milk powder at about $4-7 \mathrm{mg} / \mathrm{L}$ [1], the total dose would depend on the duration of the bottle-feeding period. Subjects born in 1954 may have incurred a larger dose compared to those born in 1955, as the contaminated milk powder was available for only 3-4 months during the summer of 1955, and

15 bottle-feeding of the youngest subjects may have overlapped to a lesser degree with this period. A difference in dose rather than differences in vulnerability may therefore explain the slightly worse outcomes among those born in 1954 than those in 1955 (Table 8).

Although all examiners were blinded as to the participants' exposure status, the 20 neurologists working for the hospitals may well have been aware of the exposure status of the participants. However, other tests would likely be more robust to this possible observer bias.

In conclusion, the present study indicates that adults who suffered arsenic poisoning during infancy reveal neuropsychological dysfunctions, while 
neurophysiological testing failed to show clear abnormalities. Unfavorable neuropsychological outcomes were present in participants without known disabilities or abnormal mental function. These findings support the notion that developmental arsenic neurotoxicity is likely to result in permanent changes in brain functions. Relatively high

5 exposures to arsenic still occur, e.g., through drinking water in West Bengal, India, and Bangladesh, where arsenic concentrations in water can reach $1-2 \mathrm{mg} / \mathrm{L}[13,25]$, i.e., similar to the levels in prepared milk using the Morinaga milk powder. The present findings therefore support the notion that prevention of early-life exposures to such substances should be vigorously pursued.

\section{Acknowledgments:}

We appreciate valuable support from Shoko Namio, Masanori Chikama, Eiko Hayashi, Miyoko Matsuzaki (Okayama Kyoritsu Hospital), Kazuhiko Satomi, Toshiko Murakami, Sachiko Yamamoto (Mizushima Kyodo Hospital), Makoto Imura (Imura Clinic),

15 Hiroyuki Doi, Toshihide Tsuda, Akiko Tokinobu, Saori Irie (Okayama University), and staff at both hospitals.

\section{Funding source:}

This work was supported by a Grant-in-Aid for Young Scientists (B) (No. 23790662)

20 from the Ministry of Education, Culture, Sports, Science and Technology, Japan. The sponsor has had no involvement in the study design, the collection, analysis, and interpretation of data, the writing of the report, or the decision to submit the paper for publication. 
Conflict of Interest Statement: The authors declare that they have no conflicts of interest.

5 


\section{References}

[1] M. Dakeishi, K. Murata, P. Grandjean, Long-term consequences of arsenic poisoning during infancy due to contaminated milk powder, Environ Health 5 (2006) 31 .

5 [2] P. Grandjean, P.J. Landrigan, Developmental neurotoxicity of industrial chemicals, Lancet 368 (2006) 2167-78.

[3] J.D. Hamadani, S.M. Grantham-McGregor, F. Tofail, B. Nermell, B. Fangstrom, S.N. Huda, S. Yesmin, M. Rahman, M. Vera-Hernandez, S.E. Arifeen and others, Pre- and postnatal arsenic exposure and child development at 18 months of age: a cohort study in rural Bangladesh, International journal of epidemiology 39 (2010) 1206-16.

[4] J.D. Hamadani, F. Tofail, B. Nermell, R. Gardner, S. Shiraji, M. Bottai, S.E. Arifeen, S.N. Huda, M. Vahter, Critical windows of exposure for arsenic-associated impairment of cognitive function in pre-school girls and boys: a population-based cohort study, International journal of epidemiology 40 (2011) 1593-604.

[5] E. Hamamoto, [Report of arsenic poisoning incident due to powdered milk in Okayama prefecture (in Japanese)], Department of health, Okayama prefecture, Okayama, Okayama, 1957.

20 [6] M. Harada, Minamata disease: methylmercury poisoning in Japan caused by environmental pollution, Critical reviews in toxicology 25 (1995) 1-24.

[7] Hikari Association, [Hikari Association (in Japanese)], 2015.

[8] B. Horta, C. Victor, Long-term effects of breastfeeding, A Systematic Review, World Health Organization2013. 
[9] Lafayette Instrument, Grooved Pegboard Test User Instructions Lafayette Instrument Company, IN, USA, 2002.

[10] National Research Council (U.S.). Subcommittee on Arsenic in Drinking Water., Arsenic in drinking water, National Academy Press, Washington, D.C.1999.

[11] H.L. Needleman, The future challenge of lead toxicity, Environ Health Perspect 89 (1990) 85-9.

[12] M. Ohira, H. Aoyama, [Epidemiological studies on the Morinaga powdered milk poisoning incident], Nihon eiseigaku zasshi Japanese journal of hygiene 27 (1973) 500-31.

[13] M. Rahman, M. Vahter, M.A. Wahed, N. Sohel, M. Yunus, P.K. Streatfield, S. El Arifeen, A. Bhuiya, K. Zaman, A.M. Chowdhury and others, Prevalence of arsenic exposure and skin lesions. A population based survey in Matlab, Bangladesh, Journal of epidemiology and community health 60 (2006) 242-8.

15 [14] J.L. Rosado, D. Ronquillo, K. Kordas, O. Rojas, J. Alatorre, P. Lopez, G. Garcia-Vargas, M. Del Carmen Caamano, M.E. Cebrian, R.J. Stoltzfus, Arsenic exposure and cognitive performance in Mexican schoolchildren, Environ Health Perspect 115 (2007) 1371-5.

[15] P.D. Sampson, A.P. Streissguth, F.L. Bookstein, R.E. Little, S.K. Clarren, P. Dehaene, J.W. Hanson, J.M. Graham, Jr., Incidence of fetal alcohol syndrome and prevalence of alcohol-related neurodevelopmental disorder, Teratology 56 (1997) 317-26.

[16] D. Sheslow, W. Adams, P. staff, Wide Range Assessment of Memory and Learning Second Edition Interpretive Report, PAR Psychological Assessment Resources, Inc., FL, USA, 2009. 
[17] A.H. Smith, C.M. Steinmaus, Health effects of arsenic and chromium in drinking water: recent human findings, Annu Rev Public Health 30 (2009) $107-22$.

[18] K. Straif, L. Benbrahim-Tallaa, R. Baan, Y. Grosse, B. Secretan, F. El Ghissassi, V. Bouvard, N. Guha, C. Freeman, L. Galichet and others, A review of human carcinogens--part C: metals, arsenic, dusts, and fibres, Lancet Oncol 10 (2009) 453-4.

[19] H. Tanaka, H. Tsukuma, A. Oshima, Long-Term Prospective Study of 6104 Survivors of Arsenic Poisoning During Infancy Due to Contaminated Milk Powder in 1955, J Epidemiol 20 (2010) 439-445.

[20] F. Tofail, M. Vahter, J.D. Hamadani, B. Nermell, S.N. Huda, M. Yunus, M. Rahman, S.M. Grantham-McGregor, Effect of arsenic exposure during pregnancy on infant development at 7 months in rural Matlab, Bangladesh, Environ Health Perspect 117 (2009) 288-93.

15 [21] M. Tolins, M. Ruchirawat, P. Landrigan, The developmental neurotoxicity of arsenic: cognitive and behavioral consequences of early life exposure, Annals of global health 80 (2014) 303-14.

[22] S.Y. Tsai, H.Y. Chou, H.W. The, C.M. Chen, C.J. Chen, The effects of chronic arsenic exposure from drinking water on the neurobehavioral development in adolescence, Neurotoxicology 24 (2003) 747-53.

[23] M. Vahter, Health effects of early life exposure to arsenic, Basic Clin Pharmacol Toxicol 102 (2008) 204-11.

[24] S. Vibol, J.H. Hashim, S. Sarmani, Neurobehavioral effects of arsenic exposure among secondary school children in the Kandal Province, Cambodia, Environmental research 137 (2015) 329-37. 
[25] O.S. von Ehrenstein, S. Poddar, Y. Yuan, D.G. Mazumder, B. Eskenazi, A. Basu, M. Hira-Smith, N. Ghosh, S. Lahiri, R. Haque and others, Children's intellectual function in relation to arsenic exposure, Epidemiology 18 (2007) 44-51.

5 [26] S.X. Wang, Z.H. Wang, X.T. Cheng, J. Li, Z.P. Sang, X.D. Zhang, L.L. Han, X.Y. Qiao, Z.M. Wu, Z.Q. Wang, Arsenic and fluoride exposure in drinking water: children's IQ and growth in Shanyin county, Shanxi province, China, Environ Health Perspect 115 (2007) 643-7.

[27] G.A. Wasserman, X. Liu, N.J. Loiacono, J. Kline, P. Factor-Litvak, A. van Geen, J.L. Mey, D. Levy, R. Abramson, A. Schwartz and others, A cross-sectional study of well water arsenic and child IQ in Maine schoolchildren, Environ Health 13 (2014) 23.

[28] G.A. Wasserman, X. Liu, F. Parvez, H. Ahsan, P. Factor-Litvak, J. Kline, A. van Geen, V. Slavkovich, N.J. Loiacono, D. Levy and others, Water arsenic exposure and intellectual function in 6-year-old children in Araihazar, Bangladesh, Environ Health Perspect 115 (2007) 285-9.

[29] G.A. Wasserman, X. Liu, F. Parvez, H. Ahsan, P. Factor-Litvak, A. van Geen, V. Slavkovich, N.J. LoIacono, Z. Cheng, I. Hussain and others, Water arsenic exposure and children's intellectual function in Araihazar, Bangladesh, Environ Health Perspect 112 (2004) 1329-33.

[30] D. Wechsler, [Technical manual for the Wechsler Adult Intelligence Scale -Third edition: Japanese translation (in Japanese)], Nihon Bunka kagakusha, Tokyo2006.

[31] World Health Organization, Environmental health criteria: 224. Arsenic and Arsenic compounds, World Health Organization, Geneva2001. 
[32] R.O. Wright, C. Amarasiriwardena, A.D. Woolf, R. Jim, D.C. Bellinger, Neuropsychological correlates of hair arsenic, manganese, and cadmium levels in school-age children residing near a hazardous waste site, Neurotoxicology 27 (2006) 210-6.

5 [33] N. Yamashita, M. Doi, M. Nishio, H. Hojo, M. Tanaka, [Recent observations of Kyoto children poisoned by arsenic tainted "Morinaga Dry Milk" (author's transl)], Nihon eiseigaku zasshi Japanese journal of hygiene 27 (1972) 364-99.

[34] T. Yorifuji, T. Tsuda, P. Grandjean, Unusual cancer excess after neonatal arsenic exposure from contaminated milk powder, J Natl Cancer Inst 102 (2010) 360-1. 